\title{
On the Study of Network Coding with Diversity
}

\author{
Zhiguo Ding, Member, IEEE, Kin K. Leung, Fellow, IEEE, Dennis L. Goeckel, Senior \\ Member, IEEE, and Don Towsley, Fellow, IEEE
}

\begin{abstract}
Recently proposed physical-layer network coding (PNC) [1] has demonstrated the promise to significantly improve the throughput of wireless networks whose links can be modeled as additive white Gaussian noise (AWGN) channels. However, the extension to multipath channels is problematic, since the technique would then require both amplitude and phase compensation at each transmitter. Phase compensation requires accurate distributed phase tracking, whereas the required amplitude compensation is even more troubling, as it leads to an inefficient system that yields no diversity even in the presence of perfect channel estimates. Here, a system that avoids these limitations is obtained by reaching up one level higher in the network hierarchy and performing distributed relay selection with cognizance of the PNC technique that we will employ at the physical layer. Since the resulting scheme will achieve a form of selection diversity, we term it "network coding with diversity" (NCD). To facilitate performance evaluation, two information-theoretic metrics, the outage and ergodic capacity, are studied. Our analytical and simulation results show that the proposed protocol achieves more robust performance and higher system throughput than comparable schemes. Finally, the proposed network coding is extended to the context of cooperative multiple access channels, which yields a new cooperative protocol with larger outage and ergodic capacity compared with existing transmission schemes.
\end{abstract}

\section{INTRODUCTION}

Network coding has emerged as a potentially powerful tool in the design of communication networks and has been widely studied since its introduction in [2]. Unlike traditional approaches to error control coding in networks, where coding was performed at the edges (i.e. "end-to-end") or on individual packets on a given link, network coding employs intermediate nodes to combine and code packets. Originally considered extensively in the context of wired communications, there has recently been extreme interest in applying network coding to wireless communication scenarios [3]-[6]. In fact, the broadcast nature of

Research was sponsored by US Army Research laboratory and the UK Ministry of Defence and was accomplished under Agreement Number W911NF-06-3-0001. The views and conclusions contained in this document are those of the authors and should not be interpreted as representing the official policies, either expressed or implied, of the US Army Research Laboratory, the U.S. Government, the UK Ministry of Defense, or the UK Government. The US and UK Governments are authorized to reproduce and distribute reprints for Government purposes notwithstanding any copyright notation hereon.

Zhiguo Ding is with Dept. of Communication Systems, Lancaster University, Lancaster, LA1 4WA, UK. Kin K. Leung is with Dept. of Electrical and Electronic Engineering, Imperial College, London, SW7 2BT, UK. Dennis L. Goeckel is with Dept. of Electrical and Computer Engineering, University of Massachusetts, Amherst, MA, US. Don Towsley is with Dept. of Computer Science, University of Massachusetts, Amherst, MA, US. 
the wireless channel allows for the exploitation of some of the particular features of network coding. The prototypical framework is that of two wireless transceivers exchanging information through a relay that lies geographically between them [7]. With standard network coding, the two transceivers each employ one time slot to transmit a packet to the relay in a conventional time-division multiple access (TDMA) scheme. Next, the relay takes the exclusive-or of these two packets and broadcasts the result during the third time slot. Armed with the packet it sent to the relay, each of the transceivers can then recover the data originating at the other relay, with the network having only used three slots rather than the traditional four [8].

Physical-layer network coding (PNC), as proposed in [1], is able to be even more efficient by reaching down into the physical layer. In particular, when two transceivers wish to communicate through an intermediate relay and the intervening channels can be assumed to be additive white Gaussian noise (AWGN), accurate distributed transmitter phase compensation, if achievable, allows the PNC scheme to have both transceivers transmit simultaneously during a single time slot. Rather than attempting to decode both of the packets, the relay only decodes the exclusive-or of the packets, which it then broadcasts during the second time slot. As in the network coding example above, each of the transceivers is then able to decode the information sourced at the other transceiver, but now the network only required two time slots for such an information exchange.

Hence, physical-layer network coding provides an idea with the potential for significant throughput gains in the wireless environment. However, due to the large scale path loss and multi-path fading generally encountered on wireless links [9], there are significant problems that need to be overcome. In particular, the key step of PNC is to decode the sum received by the relay. To accomplish such, the received signals from the two transceivers must be both precisely phase-matched and have identical received powers. This requires not only that each of the transmitters have precise (amplitude and phase) channel estimates, but also that each of the transceivers performs a form of pre-equalization - a gain compensation to invert the amplitude of the intervening channel from that transceiver to the relay. Whereas the former issue is problematic due to implementation difficulty, the latter is problematic because channel inversion is well-known to be quite inefficient. In particular, under standard frequency-nonselective Rayleigh fading channel assumptions, the average transmission power of the PNC scheme given by $\int_{0}^{\infty} \frac{1}{x} f_{X}(x) d x \rightarrow \infty$, where $x=$ is exponentially distibuted and $f_{X}(x)$ is the density function of $x$, is unbounded.

The main aim of this paper is to design a new form of network coding which can exploit the core idea of PNC to realize its significant throughput gain, but which avoids the difficult synchronization contraints and large transmission power required to realize the pre-equalization. The solution we propose is arrived at by viewing the problem more generally in the overall network context. In particular, rather than assuming 
that we are tasked with using a pre-selected relay, we instead focus on exchanging the messages from the two transceivers in a dense network where multiple relays are available for selection as the intermediate relay. By proper distributed relay selection, not only is the throughput gain of PNC realized, but a form of multi-user diversity is provided. This leads to quite promising results in terms of outage and ergodic capacity, as demonstrated here.

The contributions of this paper are as follows. First, a distributed relay selection strategy is provided that selects the relay whose resulting wireless links are best suited for physical-layer network coding. The operation of the distributed strategy will be similar to that of the strategy in [10], and our focus here is how to determine the appropriate metric for relay selection in the context of PNC. Second, an exact expression for the outage capacity for the proposed NCD, as well as a simplified approximation for the high signal-to-noise (SNR) region, are developed. Third, upper and lower bounds on the ergodic capacity are provided for the NCD scheme, and it is subsequently shown that these bounds are relatively tight. Finally, the proposed network coding with diversity is extended to the context of cooperative networks. In particular, a new protocol is proposed in this paper for cooperative multiple access channels (CMA) by exploring the features of network coding. It is well known that many existing cooperative protocols, such as those of [11] and [12], can yield large outage capacity, but suffer a loss in ergodic capacity compared with direct transmission. By exploring the throughput merit of network coding, the proposed CMA can achieve larger ergodic capacity than existing cooperative protocols and direct transmission, which has not been reported before.

This paper is organized as follows. The proposed network coding protocol is described in Section II. For performance evaluation, two types of information-theoretic metrics, outage and ergodic capacity are developed in Section III, and numerical results are shown in Section IV for performance comparison. Then the proposed network coding with diversity is extended to the context of cooperative multiple access channels in Section V. Finally, concluding remarks are given in Section VI.

\section{Protocol Description}

Consider a sensor network with $N+2$ nodes, where two source nodes try to exchange information with the help of the $N$ relaying nodes. As shown in Fig. 1, the information exchange consists of two stages or time slots. During the first stage, both source nodes broadcast their information to the whole network simultaneously. Unlike the PNC in [1], our proposed protocol does not rely on the assumption of precise phase synchronization ${ }^{1}$. And note that no mechanism of channel pre-equalization is required at the transmitters here. The transmitted signals arrive the relays corrupted by additive Gaussian noise,

\footnotetext{
${ }^{1}$ Although we do need time synchronization, the use of time division duplex systems can ensure that time synchronization is a minor difficulty compared with phase synchronization.
} 
large-scale path loss, Raleigh fading distortion, and inter-channel interference. Hence at the first time slot, the observation of the relay $R_{n}$ can be denoted as

$$
y_{R_{n}}=\sqrt{P} h_{1 R_{n}} s_{1}+\sqrt{P} h_{2 R_{n}} s_{2}+w_{R_{n}},
$$

where $P$ is the source transmission power, $s_{i}$ denotes the unit-power signal transmitted from the source $i$, $w_{R_{n}}$ denotes the additive Gaussian noise with power $P_{w}$, and $h_{i R_{n}}$ denotes the gain from the source $i$ to the relay $R_{n}$. We employ a propagation model which includes path loss, shadow fading and frequencynonselective Rayleigh fading [13]-[15], and can be modeled as

$$
h_{i R_{n}}=\frac{g_{i R_{n}}}{\sqrt{d_{i R_{n}}^{\alpha}}},
$$

where $d_{i R_{n}}$ denotes the distance between the source $i$ and the relay $R_{n}, \frac{1}{d_{i R_{n}}^{\alpha}}$ depicts the large-scale behavior of the channel gain, $\alpha$ is the path loss exponent and $g_{i R_{n}}$ captures the channel fading characteristics due to the rich scattering environment.

By using training symbols, it is reasonable to assume that each relay can obtain the knowledge of two the incoming channels, $h_{1 R_{n}}$ and $h_{2 R_{n}}$ (i.e. we make the standard assumption that the receiveas have channel state information). Due to the symmetry of time division duplex systems, the incoming channel and the return channel are assumed to be symmetric, $h_{i R_{n}}=h_{R_{n} i}$. Hence it is safe to conclude that each relay node will have the access to its local channel information without employing too much overhead. By using such local channel information, a distributed strategy of relay selection can be carried out to ensure the quality of the relayed transmission, where the detailed discussion of the strategy will be provided at the end of this section. Consider that the node $R$ has been chosen as the best relay. To simplify the notations denote the channels between the two sources and the best relay as $h_{1}$ and $h_{2}$, respectively.

During the second stage, the best relay employs the amplify-forward strategy and broadcasts the compressed mixture, $\frac{\sqrt{P} h_{1} s_{1}+\sqrt{P} h_{2} s_{2}+w_{R}}{\sqrt{P\left|h_{1}\right|^{2}+P\left|h_{2}\right|^{2}+P_{w}}} \sqrt{P}$, to the two source nodes. Since $s_{i}$ is the information known for source $i$, this part can be removed from the observation at the source $i$, which yields

$$
y_{i}=\frac{\sqrt{P} h_{i}}{\sqrt{P\left|h_{1}\right|^{2}+P\left|h_{2}\right|^{2}+P_{w}}}\left(\sqrt{P} h_{j} s_{j}+w_{R}\right)+w_{i},
$$

where $w_{i}$ denotes the additive noise at the source $i$. After algebraic manipulations, the mutual information that the source $i$ can receive from the source $j$ can be shown as

$$
\mathcal{I}_{i j, N C D}=\log \left[1+\frac{\rho^{2}\left|h_{1}\right|^{2}\left|h_{2}\right|^{2}}{2 \rho\left|h_{i}\right|^{2}+\rho\left|h_{j}\right|^{2}+1}\right], \forall \quad i \neq j \quad \& \quad i, j \in[1,2],
$$

where $\rho=P / P_{w}$ denotes the signal-to-noise ratio. As a comparable scheme, the mutual information achieved by the direct transmission scheme is

$$
\mathcal{I}_{D R}=\log \left[1+\rho|h|^{2}\right]
$$

where $h=\frac{g}{\sqrt{d_{12}^{\alpha}}}$ and $d_{12}$ is the distance between the two sources. 


\section{A. Physical Network Coding}

It is difficult to extend physical-layer network coding (PNC) to the multipath environment, which is the key motivation for this paper. However, we desire some logical extension as a standard of comparison for our proposed scheme, and thus we employ the scheme from [1] with a few modifications that make it functional. In particular, we assume that the PNC scheme of [1] is able to obtain perfect transmitter channel state information (CSI) to do its required distributed phase synchronization, but we also assume that it uses such CSI to do pre-equalization of the amplitude. Obviously, one immediately imagines that such pre-equalization will lead to inefficient signaling and loss of diversity gain, as noted above, but it is required for the operation of the scheme. Note that the extended scheme is only one of operable solutions to extend PNC to multipath fading environments, and its purpose is to provide a comparable scheme for the proposed transmission scheme.

If the pre-equalization is perfect, the observation at the relay can be written as

$$
y_{R}=\sqrt{P} s_{1}+\sqrt{P} s_{2}+w_{R}
$$

which will then be broadcast to the two source nodes. To be precise, the PNC scheme proposed in [1] utilizes the decode-forward strategy, where the received mixture is decoded and mapped to a particular constellation. As discussed in [11], the use of decode-forward or amplify-forward strategy only results in a slight difference for the outage capacity and diversity order. Hence, to facilitate analytical analysis, an amplify-forward version of the original PNC scheme is proposed here.

Unlike the first stage, pre-equalization at the second stage will be impossible since different destination node requires different equalizer coefficients. Hence, the broadcasted mixture will arrive the receivers corrupted by path loss and fading distortion. After removing the known information, the source $i$ will observe

$$
y_{i}=\frac{\sqrt{P} h_{i}}{\sqrt{2 P+P_{w}}}\left(\sqrt{P} s_{j}+w_{R}\right)+w_{i},
$$

whose mutual information can be shown as

$$
\mathcal{I}_{i j, P N C}=\log \left[1+\frac{\rho^{2}\left|h_{i}\right|^{2}}{\rho\left|h_{i}\right|^{2}+2 \rho+1}\right], \forall \quad i \neq j \quad \& \quad i, j \in[1,2] .
$$

Comparing (4) and (8), one observation is that the proposed NCD can yield larger mutual information than the modified PNC scheme. Considering the large SNR region, it can be expected that both mutual information can be written as $\log (1+\rho x)$, where $x=\frac{\left|h_{1}\right|^{2}\left|h_{2}\right|^{2}}{2\left|h_{i}\right|^{2}+\left|h_{j}\right|^{2}}$ for NCD and $x=\frac{\left|h_{i}\right|^{2}}{\left|h_{i}\right|^{2}+2}$ for PNC. Provided that there are large number of relaying candidates, the use of relay selection can make it possible that $x \geq 1$ for NCD. But $x$ will be always less than 1 for PNC with or without relay selection, which illustrates that wireless diversity gain is not fully utilized (as expected) by PNC. This is due to the fact that 
PNC must treat the fading as a negative factor and uses the technique of pre-equalization to pre-cancel channel fading. In the following sections, we will provide more detailed analytical and numerical results to compare the performance of the two network coding schemes.

\section{B. A distributed strategy of relay selection}

The distributed strategy is carried out at the medium access layer to select the best relaying node. To be specific, the backoff period of each relay for carrier sensing is inversely proportional to the quality of its local channel information, where the detailed description for such a distributed relay-selection strategy can be found at [10]. Our focus here is how to determine the criterion for the link quality, which is crucial to the implementation of the selection strategy. From (4), it is observed that the two destinations have different preferences. Fortunately, these two preferences do not tend to contradict each other. The relay whose channels $\mathcal{I}_{12, N C D}$ also has channels that yield a large value for $\mathcal{I}_{21, N C D}$, if not exactly the maximum.Although we currently do not have a formal proof for this claim, which appears to be difficult to establish with any reasonable utility, simulations show that it is the case, as is provided Table I.

Consider two types of parameter setups. There are $N=2$ relaying candidates for the first setup and $N=10$ relays for the second setup. The channel factor is generated according to (2) where the distance between the relay $R_{n}$ and the source $i, d_{i R_{n}}$, is random chosen from the range $[1 \mathrm{~m} 10 \mathrm{~m}]$. For each experiment, the best relay is chosen according to the following criterion

$$
\frac{\left|h_{1}\right|^{2}\left|h_{2}\right|^{2}}{2 \rho\left|h_{1}\right|^{2}+\rho\left|h_{2}\right|^{2}+1}
$$

which maximizes the value of $\mathcal{I}_{12, N C D}$. Then using this chosen relay, the mutual information for the second source, $\mathcal{I}_{21, N C D}$, is calculated. Ergodic capacity is then obtained by implementing Monte Carlo simulation. Since such the chosen relay is suboptimal for the second source, it can be expected that there will some performance penalty for the capacity of the second source. However, as shown in Table I, such performance loss due to the use of the suboptimal relay is neglectable.

\section{Information-Theoretic Metrics: OUtAGE AND ERgodic CAPACITY}

In this section, we aim to study two different information-theoretic metrics in order to evaluate the performance of the proposed network coding protocol. The first is termed as outage capacity, or outage probability, which shows the robustness of a communication system and measures the data rate that can be supported with a certain error probability. The other is termed as ergodic capacity, which is intended to measure the long-term system throughput and obtained by averaging the mutual information over all possible channel realizations. In the following, the expression of the two kind of capacity will be developed for the proposed network coding protocol and compared with some existing schemes. 


\section{A. Outage capacity}

First the definition of the outage capacity is given here.

Definition 1: $\alpha \%$ outage capacity is the data rate that can be supported with $\alpha \%$, i.e.,

$$
P(\mathcal{I}<R) \leq \alpha \% \text {. }
$$

From its definition, the outage capacity can be obtained from the cumulative distribution function (CDF) of the mutual information $\mathcal{I}_{N C D}$. Provided the relay selection strategy is not applied, a random relay $R_{n}$ is used for relaying. From (4), the mutual information achieved by such a random relay can be written as

$$
\mathcal{I}_{n}=\log \left[1+\frac{1}{2} \frac{x_{n} y_{n}}{x_{n}+y_{n}+1}\right],
$$

where $x_{n}=2 \rho\left|h_{1 R_{n}}\right|^{2}$ and $y_{n}=\rho\left|h_{2 R_{n}}\right|^{2}$. Due to the system symmetry, here we only focus on the mutual information $\mathcal{I}_{12, N C D}$ and the subscription $\{12\}$ will be omitted to simplify notations.

As can be seen from (2), $\left|h_{i R_{n}}\right|^{2}$ will be exponentially distributed with the parameter $d_{i R_{n}}^{\alpha}$. So $x_{n}$ will be exponentially distributed with the parameter $\lambda_{x n}=\frac{d_{1 R_{n}}^{\alpha}}{2 \rho}$ and $y_{n}$ will be exponentially distributed with the parameter $\lambda_{y n}=\frac{d_{2 R_{n}}^{\alpha}}{\rho}$. For the simplicity of analytical development, similar to [15], it is assumed the the distance between relays is far less than the distance $d_{i n}$, which implies that $d_{i n}=d_{i k}=d_{i}, \forall n \neq k$. Hence the $N$ mutual information will be identically independent distributed, which can be ordered as

$$
\mathcal{I}_{(1)} \leq \mathcal{I}_{(2)} \leq \cdots \leq \mathcal{I}_{(N)}
$$

And the relay corresponding to the largest value of the mutual information will be chosen according to the description of relay selection, which means that $\mathcal{I}_{(N)}=\mathcal{I}_{N C D}$.

Hence it is desirable to first find the density function of $\mathcal{I}_{n}$, and then the order statistics can be applied to find the CDF of $\mathcal{I}_{(N)}$. Define $z_{n}=\frac{x_{n} y_{n}}{x_{n}+y_{n}+1}$, and it is desirable to find the distribution function of $z_{n}$, which is provided in the following lemma.

Lemma 1: Provided that $x$ is exponentially distributed with $\lambda_{x}$ and $y$ is exponentially distributed with $\lambda_{y}$, the CDF of the variable $z=\frac{x y}{x+y+1}$ can be shown as

$$
P(\mathbf{z}<z)=1-e^{-\left(\lambda_{x}+\lambda_{y}\right) z} \sqrt{4 \lambda_{x} \lambda_{y} z(z+1)} \mathbf{K}_{1}\left(\sqrt{4 \lambda_{x} \lambda_{y} z(z+1)}\right)
$$

where $\mathbf{K}_{1}(x)$ is the modified bessel function of the second kind.

Proof: See Appendix.

From Lemma 1, it is interesting to remark that Lemma 1 in [11] can be easily proved by using the following approximation

$$
\mathbf{K}_{1}(x) \underset{x \rightarrow 0}{\approx} \frac{1}{x}
$$

Furthermore, we can have the following theorem about the outage probability of the proposed protocol. 
Theorem 2: The exact expression of the outage probability for the proposed NCD can be shown as

$$
P\left(\mathcal{I}_{N C D}<R\right)=\left(1-e^{-\left(\lambda_{x n}+\lambda_{y n}\right) \gamma} \sqrt{\theta_{n}} \mathbf{K}_{1}\left(\sqrt{\theta_{n}}\right)\right)^{N} .
$$

where $\theta=4 \lambda_{x n} \lambda_{y n} \gamma(\gamma+1)$ and $\gamma=2\left(2^{R}-1\right)$. And, at high SNR region, the outage probability of the NCD can be shown as

Proof: See Appendix.

$$
P\left(\mathcal{I}_{N C D}<R\right) \approx \frac{\left[\left(d_{1}^{\alpha}+2 d_{2}^{\alpha}\right)\left(2^{R}-1\right)\right]^{N}}{\rho^{N}} .
$$

From Definition 1, the outage capacity of the proposed protocol can be easily calculated by using Theorem 2. Furthermore, an important conclusion from Theorem 2 is that the proposed network coding protocol can achieve the diversity gain $N$, which is due to its superior ability to explore the diversity of wireless multipath fading. Provided the existence of multiple relays, it is possible that we can find one relay which has good connection with both two sources, which yields the so-called multi-user/relay diversity. As a comparable scheme, the PNC scheme can achieve the outage probability as

$$
\begin{aligned}
P\left(\mathcal{I}_{P N C}<R\right) & =1-\exp \left\{-2 \lambda_{i} \frac{2^{R}-1}{\rho} /\left(1-\frac{2^{R}-1}{\rho}\right)\right\} \\
& \approx d_{i}^{\alpha} \frac{2^{R}-1}{\rho},
\end{aligned}
$$

and the direct transmission scheme can have the outage probability

$$
P\left(\mathcal{I}_{D R}<R\right) \approx d_{12}^{\alpha} \frac{2^{R}-1}{\rho},
$$

where $\lambda_{i}=d_{i}^{\alpha}$ and the last equality of (15) follows from the approximation $e^{-x} \underset{x \rightarrow 0}{\approx} 1-x$. As can be seen from (15) and (16), both the PNC scheme and the direct transmission can only achieve the diversity gain 1. Hence, provided that $S N R$ is large enough, it is safe to conclude that the proposed NCD scheme can have larger outage capacity than the PNC scheme, which demonstrates that the proposed NCD is much more robust than the two comparable schemes.

Since the addressed scenario can be seen as a special case of distributed multiple-input multiple-output system, to which the diversity-multiplexing tradeoff is also of interest. We recall the definition of the diversity gain and multiplexing gain from [16], [17] as

$$
d \triangleq-\lim _{\rho \rightarrow \infty} \frac{\log \left[P_{e}(\rho)\right]}{\log \rho}, \quad r \triangleq \lim _{\rho \rightarrow \infty} \frac{R(\rho)}{\log \rho},
$$

where $P_{e}$ is the ML probability of detection error and $R$ is the data rate in bits per symbol period. By using Theorem 2, we can obtain the following corollary for the tradeoff.

Corollary 3: The diversity-multiplexing tradeoff for the proposed network coding protocol can be expressed as

$$
d(r)=N(1-r) .
$$


Proof: See Appendix.

Again Corollary 3 confirms our claim that the proposed NCD protocol can achieve the diversity gain order $N$. Another important remark implied by Corollary 3 is that the NCD protocol can have the same multiplexing gain as the direct transmission scheme, whereas many exiting diversity schemes, such as the ones in [11], [18], can only achieve the fraction of the multiplexing gain 1. Such property is valuable because higher multiplexing gain typically yields higher ergodic capacity which will be discussed in detail in the following subsection.

\section{B. Ergodic Capacity}

Recall that having larger outage capacity does not necessarily ensure a system to have larger ergodic capacity. For example, cooperative protocols typically yield the larger outage capacity than direct transmission, but smaller ergodic capacity, which is due to that relaying transmission could cost the extra use of bandwidth resource. Recall that the proposed NCD only requires two time slots to accomplishing information exchanging, the same amount bandwidth resource required by the direct transmission scheme. Hence an intuition tells us that the proposed NCD protocol should be able to avoid the loss of ergodic capacity, which will be confirmed by the following developed results. First the definition for the ergodic capacity can be written as

Definition 2: Ergodic capacity is the long-term data rate that a system can support, i.e.,

$$
\mathcal{C}_{e}=\int_{0}^{\infty} \mathcal{I} f_{\mathcal{I}}(\mathcal{I}) d \mathcal{I},
$$

where $f_{\mathcal{I}}(\cdot)$ is the probability density function $(\mathrm{PDF})$ of the mutual information $\mathcal{I}$.

Hence the calculation of the ergodic capacity requires the expression of the PDF of the mutual information $\mathcal{I}_{N C D}$. As can be seen from (11), the CDF of the $\mathcal{I}_{n}$ includes the bessel function, which makes that the exact expression of its PDF difficult to be obtained. So it will be valuable for performance evaluation to develop the lower and upper bounds of the ergodic capacity. As a first step, the following theorem provides the upper and lower bounds for the CDF of the variable $z_{n}$.

Lemma 4: Provided that $x$ is exponentially distributed with $\lambda_{x}$ and $y$ is exponentially distributed with $\lambda_{y}$, the CDF of the variable $z=\frac{x y}{x+y+1}$ can be bounded as

$$
1-e^{-\left(\lambda_{x}+\lambda_{y}\right) z} \leq P(\mathbf{z}<z) \leq 1-e^{-\left(\sqrt{\lambda_{x}}+\sqrt{\lambda_{y}}\right)^{2} z}
$$

Proof: See Appendix.

It can be expected that there are many other forms of bounds for $P(\mathbf{z}<z)$, however, the two bounds in Lemma 4 are chosen because of the similarity of their expression. It is interesting to observe that both two bounds are exponentially distributed, which will simplify the the following derivation. The development 
of the upper and lower bounds of the ergodic capacity will be exactly same, just with different parameters. By using Lemma 4, we can obtain the following theorem about the upper and lower bounds of the ergodic capacity.

Theorem 5: The ergodic capacity of the proposed network coding protocol can be bounded as the following

$$
\log \rho-2 \log \left(\sqrt{\frac{d_{1}^{\alpha}}{2}}+\sqrt{d_{2}^{\alpha}}\right)+D \leq \mathcal{C}_{N C D} \leq \log \rho-\log \left(\frac{d_{1}^{\alpha}}{2}+d_{2}^{\alpha}\right)+D
$$

where $\mathbf{C}$ denotes the Euler's constant and $D=\sum_{k=0}^{N-1} C_{N}^{k+1}(-1)^{k+1} \log (k+1)-\mathbf{C} \log e-1$ is a constant not related with SNR.

Proof: See Appendix.

It can be easily to be obtained that the ergodic capacity for direct transmission can be expressed as

$$
\begin{aligned}
\mathcal{C}_{D R} & =\int_{0}^{\infty} \log (1+x) \lambda e^{-\lambda x} d x \\
& =e^{\lambda} \log e[-\mathbf{E i}(-\lambda)] \\
& \approx-\log e[\mathbf{C}+\ln (\lambda)]=\log \rho-\log d_{12}^{\alpha}-\mathbf{C} \log e
\end{aligned}
$$

where $\lambda=\frac{d_{12}^{\alpha}}{\rho}, \operatorname{Ei}(\cdot)$ denotes the exponential-integral function and $d_{12}$ denotes the distance between the two source nodes.

When SNR approximates to infinity, both two schemes will achieve the same ergodic capacity $\mathcal{C}_{D R} \approx$ $\mathcal{C}_{N C D} \approx \log (\rho)$. However, for moderate SNR, there will a constant difference between the ergodic capacity achieved by two schemes, which can be bounded as

$$
\mathcal{C}_{N C D}-\mathcal{C}_{D R} \geq \log d_{12}^{\alpha}-2 \log \left(\sqrt{\frac{d_{1}^{\alpha}}{2}}+\sqrt{d_{2}^{\alpha}}\right)+\sum_{k=0}^{N-1} C_{N}^{k+1}(-1)^{k+1} \log (k+1)-1 .
$$

Assume that the all relays are situated at the middle of the two sources, we can have $d_{12}=2 d_{i}$, and then the difference of the two types of capacity can be simplified as

$$
\mathcal{C}_{N C D}-\mathcal{C}_{D R} \geq \alpha-2 \log \left(\sqrt{\frac{1}{2}}+1\right)+\sum_{k=0}^{N-1} C_{N}^{k+1}(-1)^{k+1} \log (k+1)-1 .
$$

Note that for $N \geq 2$, we can have $\sum_{k=0}^{N-1} C_{N}^{k+1}(-1)^{k+1} \log (k+1) \geq 1$. Recall the typical value of the path loss parameter $\alpha$ is larger than 2 . Hence we can obtain

$$
\mathcal{C}_{N C D}-\mathcal{C}_{D R} \geq 0
$$

which demonstrates that the proposed NCD protocol can always achieve larger ergodic capacity than the direct transmission scheme. Unfortunately, the closed-form expression of the ergodic capacity for the PNC protocol can not be found, but we will provide simulation results for detailed comparison in the next section. 


\section{NuMERicAl RESUlts}

In this section, we will provide detailed simulation results to compare the performance of the proposed network coding protocol with the two compared schemes, the direct transmission and modified physical network coding scheme. As discussed in Section II-A, the adopted PNC scheme is only one of feasible solutions to extend PNC to wireless fading environments, and its purpose is to serve as a comparable scheme and facilitate performance evaluation. Since both the outage capacity and ergodic capacity are equally important information-theoretic measures, we will provide two subsections in the following for the two types of capacity respectively.

\section{A. Outage Capacity}

Consider that the two sources are separated with the distance $d_{12}=2 \mathrm{~m}$, and all relays are situated at the center of the two sources, $d_{1 n}=d_{2 n}$, for $n \in[1, \cdots, N]$. The path loss factor is set as $\alpha=2$. Fig. 2 shows the outage capacity of the three transmission schemes at different SNR. As can be seen from the two sub-figures, the proposed protocol can achieve larger outage capacity than the two compared schemes. For example, at $\mathrm{SNR}=20 \mathrm{~dB}, 10 \%$ outage capacity of the proposed scheme with the use of two relays is $3.5 \mathrm{bits} / \mathrm{s} / \mathrm{Hz}$ whereas the physical network coding achieves only $2.5 \mathrm{bits} / \mathrm{s} / \mathrm{Hz}$ and the direct transmission scheme achieves even less, only 1.9 bits/s/Hz (see Fig. 2). The capacity achieved by the proposed network coding can be furthermore improved with the use of more relays. In Fig. 2, the results obtained by using the developed analytical formulations are also shown to be close to the Monte-Carlo simulation results, which demonstrates the accuracy of our developed analytical results.

When the distance of the two sources is enlarged, the performance of all schemes will be reduced as expected. As shown in Fig. 3, it is interesting to observe that the PNC can achieve larger outage capacity than the proposed NCD at low SNR. But by increasing SNR, eventually the PNC will be outperformed by the proposed scheme. The reason for this phenomenon can be clarified as the following. Recall from Theorem 1, the outage probability of the proposed protocol is proportional to $\frac{1}{\rho^{N}}$ multiplied by a constant. For low SNR, the constant multiplier could dominate the outage probability, and hence the difference of the diversity gain is not much important. But at the high region of SNR, $\frac{1}{\rho^{N}}$ becomes the dominant factor, and eventually a scheme with higher diversity gain should achieve large outage capacity than the one with lower diversity gain.

\section{B. Ergodic Capacity}

Recall that the ergodic capacity provides us the long-term throughput a system can achieve. In Fig. 4, the ergodic capacity achieved by the three schemes is shown as a function of SNR. As can be seen from the two 
sub-figures, the proposed protocol can achieve larger ergodic capacity than the direct transmission scheme at all SNR and the source separation distance, which is consistent to the analytical results developed at the end of Section III. One interesting observation from Fig. 4 is that the physical network coding is possible to outperform the proposed protocol for the large distance $d_{12}$. One reason for this is that large scale path loss becomes the dominant factor compared with the small scale multipath fading. But a more important reason is that the PNC utilizes the higher transmission power due to the use of the pre-equalization technology. For example, the signal transmitted by the source $i$ can be written as $\frac{\sqrt{P d_{i}^{\alpha}} s_{i}}{h_{i}}$, which means the average transmission power of the source is $d_{i}^{\alpha}$ times of the power used by the proposed protocol. Furthermore, Fig. 5 is provided to show the relationship of the two developed bounds and the actual value of the ergodic capacity for the proposed protocol. As can be seen from the figure, the capacity of the propose scheme is accurately bounded within the area defined by the upper and lower bounds.

\section{PNC with Diversity}

The results provided previously show that our proposed can outperform the modified PNC and direct transmission schemes. Note that the discussed relay selection can be also applied to the PNC scheme. Hence it is an interesting question how the proposed network coding protocol compares with the PNC scheme with diversity, which is answered by Fig. 6. Recall that the mutual information for the PNC scheme can be written as

$$
\mathcal{I}_{i j, P N C}=\log \left[1+\frac{\rho^{2}\left|h_{R_{n} i}\right|^{2}}{\rho\left|h_{R_{n} i}\right|^{2}+2 \rho+1}\right], \forall \quad i \neq j \quad \& \quad i, j \in[1,2] .
$$

which implies that $\mathcal{I}_{12, P N C}$ prefers the relay with the largest value of $\left|h_{R_{n} 1}\right|^{2}$, but $\mathcal{I}_{21, P N C}$ prefers the relay with the largest value of $\left|h_{R_{n} 2}\right|^{2}$. As one possible tradeoff, we choose the relay $R_{n}$ maximizing $\frac{\left|h_{R_{n} 1}\right|^{2}\left|h_{R_{n}}\right|^{2}}{\left|h_{R_{n}}\right|^{2}+\left|h_{R_{n}}\right|^{2}}$ during the simulation, which will pick up the relay with the largest value of both two $\left|h_{R_{n} i}\right|^{2}$ with constraint $\left|h_{R_{n} 1}\right|^{2} /\left|h_{R_{n} 2}\right|^{2}=1$. As can be seen from the figure, the performance of the PNC scheme could be improved by increasing the number of relaying candidates. However, the proposed scheme can achieve better performance than the PNC with diversity, specially for the large number of relays $N$. For different source separation distance, the PNC scheme is possible to outperform the proposed scheme. But the PNC scheme will always suffer the following two drawbacks. Firstly it requires the perfect synchronization. Furthermore, the PNC scheme typically requires larger transmission power than our proposed protocol, specially for the long distance between the two sources.

\section{Extension to Cooperative Multiple Access Channels}

The network coding protocol discussed previously is only suitable for the scenario that two sources are exchanging information with each other. In this section the idea of the network coding with diversity 
is extended to the cooperative multiple access channels. Consider a centralized communication system with 2 single-antenna sources, one relaying node and a central node, where the described protocol can be extended to the multi-user scenarios straightforward. Examples of such communication scenario could be a cellular system with multiple users communicating to a base station, or a sensor network controlled by a data-fusion center.

Similar to the scheme discussed previously, the cooperative transmission consists of two stages. During the first stage, both two sources are broadcasting their messages simultaneously, where both the relay and the common destination are listing. For the addressed centralized system, the strategy of relay selection can be easily implemented, which will be omitted due to the space limitation. The question of how to choose the criterion for relay quality comparison will be discussed later. Denote $R$ as the relay which is chosen as the best relaying. Hence the observation at the best relay can be written as

$$
y_{r}=h_{1 R} s_{1}+h_{2 R} s_{2}+n_{R},
$$

and the observation at the central node is

$$
y_{D, 1}=h_{1 D} s_{1}+h_{2 D} s_{2}+n_{1},
$$

where $h_{i x}$ denotes the coefficient of the channel from the source $i$ to the node $x$.

During the second stage, the relay will compress its observation and forward it to the central node. Hence at the second time slot, the the destination will receive

$$
y_{D, 2}=\frac{h_{R D}}{\beta} y_{r}+n_{2} .
$$

where $\beta=\sqrt{\left|h_{1, R}\right|^{2}+\left|h_{2, R}\right|^{2}+1 / \rho}$ is the factor to meet the power constraint. And combining (27) and (28), the signal model for such cooperative multiple access can be expressed as

$$
\mathbf{y}_{D}=\mathbf{H} \mathbf{s}+\mathbf{n}_{D},
$$

where $\mathbf{y}_{D}=\left[\begin{array}{ll}y_{D, 1} & y_{D, 2}\end{array}\right]^{T}, \mathbf{n}_{D}=\left[\begin{array}{ll}n_{1} & n_{2}+\frac{h_{R, D}}{\beta} n_{R}\end{array}\right]^{T}$ and

$$
\mathbf{H}=\left[\begin{array}{cc}
h_{1, D} & h_{2, D} \\
\frac{h_{R, D} h_{1, R}}{\beta} & \frac{h_{R, D} h_{2, R}}{\beta}
\end{array}\right] .
$$

Provided that a symmetric system is assumed here, the mutual information each use is able to achieve can be written as

$$
\mathcal{I}_{C M A}=\frac{1}{2} \log \operatorname{det}\left[\mathbf{I}_{2}+\mathbf{H}^{H} \mathbf{C}_{\mathbf{n}}{ }^{-1} \mathbf{H}\right],
$$

where $\mathbf{C}_{\mathbf{n}}{ }^{-1}=E\left\{\mathbf{n n}^{H}\right\}=\sigma^{2}\left[\begin{array}{ll}1 & 0 \\ 0 & \alpha\end{array}\right], \alpha=1 /\left(1+\frac{\left|h_{R, D}\right|^{2}}{\beta^{2}}\right), \sigma^{2}$ is the noise power and the factor $\frac{1}{2}$ is due to the fact that communication happens in 2 successive channel uses. 
After some algebraic manipulations, the mutual information can be written as

$$
\begin{aligned}
\mathcal{I}_{C M A}= & \frac{1}{2} \log \left[1+\rho \alpha\left|h_{R, D}\right|^{2}+\rho\left(\left|h_{1, D}\right|^{2}+\left|h_{2, D}\right|^{2}\right)+\frac{\rho^{2}\left|h_{R, D}\right|^{2}}{\left|h_{1, R}\right|^{2}+\left|h_{2, R}\right|^{2}+\left|h_{R, D}\right|^{2}}\right. \\
& \left.\times\left(\left|h_{1, D}\right|^{2}\left|h_{2, R}\right|^{2}+\left|h_{2, D}\right|^{2}\left|h_{1, R}\right|^{2}-2 \mathcal{R}\left\{h_{1, D}^{*} h_{2, D} h_{1, R} h_{2, R}^{*}\right\}\right)\right]
\end{aligned}
$$

which provides the criterion for the relay quality comparison. According to Jensen's inequality, the ergodic capacity of such a system can be shown as

$$
\begin{aligned}
\mathcal{E}\left\{\mathcal{I}_{C M A}\right\} \geq & \frac{1}{2} \log \mathcal{E}\left\{\left[1+\rho \alpha\left|h_{R, D}\right|^{2}+\rho\left(\left|h_{1, D}\right|^{2}+\left|h_{2, D}\right|^{2}\right)+\frac{\rho^{2}\left|h_{R, D}\right|^{2}}{\left|h_{1, R}\right|^{2}+\left|h_{2, R}\right|^{2}+\left|h_{R, D}\right|^{2}}\right.\right. \\
& \left.\left.\times\left(\left|h_{1, D}\right|^{2}\left|h_{2, R}\right|^{2}+\left|h_{2, D}\right|^{2}\left|h_{1, R}\right|^{2}-2 \mathcal{R}\left\{h_{1, D}^{*} h_{2, D} h_{1, R} h_{2, R}^{*}\right\}\right)\right]\right\} \\
\approx & \frac{1}{2} \log \mathcal{E}\left\{\left[1+\rho \alpha\left|h_{R, D}\right|^{2}+\rho\left(\left|h_{1, D}\right|^{2}+\left|h_{2, D}\right|^{2}\right)+\frac{\rho^{2}\left|h_{R, D}\right|^{2}}{\left|h_{1, R}\right|^{2}+\left|h_{2, R}\right|^{2}+\left|h_{R, D}\right|^{2}}\right.\right. \\
& \left.\left.\times\left(\left|h_{1, D}\right|^{2}\left|h_{2, R}\right|^{2}+\left|h_{2, D}\right|^{2}\left|h_{1, R}\right|^{2}\right)\right]\right\}
\end{aligned}
$$

On the other hand, recall the signal model for direct transmission can be written as

$$
\mathbf{y}_{D}=\left[\begin{array}{cc}
h_{1, D} & 0 \\
0 & h_{2, D}
\end{array}\right] \mathbf{s}+\mathbf{n}_{D R}
$$

which results the mutual information

$$
\mathcal{I}_{D R} \geq \frac{1}{2} \log \left[1+\rho\left(\left|h_{1, D}\right|^{2}+\left|h_{2, D}\right|^{2}\right)+2 \rho^{2}\left|h_{1, D}\right|^{2}\left|h_{2, D}\right|^{2}\right]
$$

Assuming that there are sufficient candidates for relaying nodes, it is possible that we can find a relay with good-quality incoming and outgoing channels to satisfy

$$
\left|h_{1, D}\right|^{2}\left(\left|h_{2, R}\right|^{2}-\gamma\left|h_{2, D}\right|^{2}\right) \geq 0 \quad \& \quad\left|h_{2, D}\right|^{2}\left(\left|h_{1, R}\right|^{2}-\gamma\left|h_{1, D}\right|^{2}\right) \geq 0
$$

where $\gamma=1 / \frac{\left|h_{R, D}\right|^{2}}{\left|h_{1, R}\right|^{2}+\left|h_{2, R}\right|^{2}+\left|h_{R, D}\right|^{2}}$. Now combining (34), (32) and (35), with sufficient candidates of relays, we can obtain

$$
\mathcal{E}\left\{\mathcal{I}_{C M A}\right\}>\mathcal{E}\left\{\mathcal{I}_{D R}\right\}
$$

Such superior performance is due to the spectrally efficiency of the proposed CMA. The whole transmission process for the proposed scheme only requires two time slots, whereas relaying transmission for most existing cooperative schemes requires the extra use of time slots. For performance evaluation, it will be desirable to obtain the exact expression of the outage probability and ergodic capacity for the proposed protocol. However, the expression of $\mathcal{I}_{C M A}$ in (30) is quite complex, and hence the performance of the proposed CMA will be examined by using Monte-Carlo simulations.

Consider an indoor rich-scattering environment which is typically assumed by existing cooperative schemes as in [11], [12]. Hence all addressed channels can be treated as identically independent Raleigh 
distributed. The performance of the direct transmission scheme as well as the classical cooperative scheme in [11], termed as the LTW scheme, is shown as the two comparable schemes. To be fair for the comparison, the strategy of relay selection is also applied to the LTW scheme. In Fig. 7, the ergogic capacity of the three schemes is shown as a function of SNR. As can be seen from the figure, the LTW protocol can only realize a fraction of the capacity achieved by direct transmission. The reason for such phenomenon is that the LTW scheme requires the extra use of one channel use to increase the reception reliability. However, the proposed cooperative protocol does not suffer such loss of ergodic capacity as shown by the figure. The last figure, Fig. 8, shows the density function of the mutual information for the three schemes. In general, the proposed cooperative scheme can achieve the largest outage probability for any outage probability, whereas the LTW scheme can only ensure the larger capacity than direct transmission at small outage provability.

\section{CONCLUSION}

Physical layer network coding (PNC) has demonstrated the promise to provide significant throughput gains in wireless networks [1], but significant problems in extending the technique to multipath fading channels have motivated questions about its widespread utility. Here, by reaching up to higher layers of the network and selecting a relay resulting in channel characteristics matched to the PNC approach, we are able to achieve the promise of physical-layer network coding and also to couple it with multi-user diversity gains. Two information-theoretic metrics, the outage and ergodic capacity, have been evaluated for the proposed scheme. The analytical and confirming simulation results show that the proposed protocol can achieve better performance than comparable schemes. Furthermore, by using the proposed approach to network coding, a new protocol of cooperative multiple access channels has been developed, which is shown to achieve more robust performance and higher system throughput than a direct transmission scheme and a classical cooperative protocol.

\section{APPENDIX}

Proof for Lemma 1 : Recall that both two variables $x$ an $y$ are exponentially distributed. From the definition, the CDF function can be written as

$$
P(\mathbf{z}<z)=\int_{\frac{x y}{x+y+1}<z} \int_{x} \lambda^{-\lambda_{x} x} \lambda_{y} e^{-\lambda_{y} y} d x d y
$$

Since $x \geq 0$, the integral area can be separated into two parts, and we can have

$$
\begin{aligned}
P(\mathbf{z}<z)= & \int_{0}^{z} \lambda_{y} e^{-\lambda_{y} y} \int_{0}^{\infty} \lambda_{x} e^{-\lambda_{x} x} d x d y \\
& +\int_{z}^{\infty} \lambda_{y} e^{-\lambda_{y} y} \int_{0}^{\frac{z y+z}{y-z}} \lambda_{x} e^{-\lambda_{x} x} d x d y .
\end{aligned}
$$


The first part at the left side of (38) can be easily calculated as

$$
\int_{0}^{z} \lambda_{y} e^{-\lambda_{y} y} \int_{0}^{\infty} \lambda_{x} e^{-\lambda_{x} x} d x d y=1-e^{-\lambda_{y} z} .
$$

For the second part of (38), we can simplify it as

$$
\begin{aligned}
& \int_{z}^{\infty} \lambda_{y} e^{-\lambda_{y} y} \int_{0}^{\frac{z y+z}{y-z}} \lambda_{x} e^{-\lambda_{x} x} d x d y \\
= & \int_{z}^{\infty} \lambda_{y} e^{-\lambda_{y} y}\left[1-e^{-\lambda_{x} \frac{z y+z}{y-z}}\right] d y \\
= & e^{-\lambda_{y}}-\lambda_{y} e^{-\lambda_{x} z} e^{-\lambda_{y} z} \int_{0}^{\infty} e^{-\alpha t-\frac{\beta}{4 t}} d t,
\end{aligned}
$$

where $\alpha=\lambda_{y}$ and $\beta=4 \lambda_{x} z(z+1)$. Unfortunately, the closed-form of the integral in (41) does not exist, and we have to rely on the bessel function. From Eq. (3.324.1) in [19], (41) can be written as

$$
\begin{aligned}
& \int_{z}^{\infty} \lambda_{y} e^{-\lambda_{y} y} \int_{0}^{\frac{z y+z}{y-z}} \lambda_{x} e^{-\lambda_{x} x} d x d y \\
= & e^{-\lambda_{y}}-\lambda_{y} e^{-\lambda_{x} z} e^{-\lambda_{y} z} \sqrt{\frac{4 \lambda_{x} z(z+1)}{\lambda_{y}}} \mathbf{K}_{1}\left(\sqrt{4 \lambda_{x} \lambda_{x} z(z+1)}\right) .
\end{aligned}
$$

Then combining (39) and (42), the lemma can be proved after some algebraic manipulations.

Proof for Theorem 2: First the CDF of the mutual information $\mathcal{I}_{n}$ is obtained as the following

$$
P\left(\mathcal{I}_{n}<R\right)=P\left(\frac{x_{n} y_{n}}{x_{n}+y_{n}+1}<2\left(2^{R}-1\right)\right) .
$$

By using Lemma 1, we can obtain

$$
P\left(\mathcal{I}_{n}<R\right)=1-e^{-\left(\lambda_{x n}+\lambda_{y n}\right) \gamma} \sqrt{\theta_{n}} \mathbf{K}_{1}\left(\sqrt{\theta_{n}}\right)
$$

where $\theta_{n}=4 \lambda_{x n} \lambda_{y n} \gamma(\gamma+1)$ and $\gamma=2\left(2^{R}-1\right)$.

By using the order statistics, the density function of the largest value can be expressed as [20]

$$
\begin{aligned}
P\left(\mathcal{I}_{(N)}<R\right) & =\left(P\left(\mathcal{I}_{n}<R\right)\right)^{N} \\
& =\left(1-e^{-\left(\lambda_{x n}+\lambda_{y n}\right) \gamma} \sqrt{\theta_{n}} \mathbf{K}_{1}\left(\sqrt{\theta_{n}}\right)\right)^{N} .
\end{aligned}
$$

Since $\theta_{n}=4 \lambda_{x n} \lambda_{y n} \gamma(\gamma+1)$, we can have $\theta \rightarrow 0$ for $\rho \rightarrow \infty$ and a fixed value of the data rate $R$. Hence by using the approximation in (12), the CDF of the outage probability can be approximated as

$$
\begin{aligned}
P\left(\mathcal{I}_{(N)}<R\right) & \approx\left(1-e^{-\left(\lambda_{x n}+\lambda_{y n}\right) \gamma}\right)^{N} \\
& \approx \gamma^{N}\left(\lambda_{x n}+\lambda_{y n}\right)^{N}
\end{aligned}
$$


where the last inequality follows from the approximation $e^{-x} \underset{x \rightarrow 0}{\approx} 1-x$. And the high-SNR approximation of the outage capacity is proved.

Proof for Corollary 3: As pointed out in [16], [17], the optimal error probability can be tightly bounded by the outage probability, provided that the block length is long enough. So in the following, we will develop the diversity-multiplexing tradeoff by using the outage probability.

Following the similar steps in [12], [16], [17], the diversity-multiplexing tradeoff for the proposed PNC can be obtained by substituting $R=r \log \rho$ into the expression of the outage capacity (45) as

$$
P\left(\mathcal{I}_{N C D}<r \log \rho\right)=\left(1-e^{-2\left(\lambda_{x n}+\lambda_{y n}\right)\left(\rho^{r}-1\right)} \sqrt{\tilde{\theta}_{n}} \mathbf{K}_{1}\left(\sqrt{\tilde{\theta}_{n}}\right)\right)^{N} .
$$

where $\tilde{\theta}_{n}=8 \lambda_{x n} \lambda_{y n}\left(\rho^{r}-1\right)\left(2\left(\rho^{r}-1\right)+1\right)$. So from the definition of the diversity gain, we can have

$$
\begin{aligned}
d(r) & =-\lim _{\rho \rightarrow \infty} \frac{\log P\left(\mathcal{I}_{(N)}<r \log \rho\right)}{\log \rho} \\
& =-\lim _{\rho \rightarrow \infty} \frac{\log \left(1-e^{-2\left(\lambda_{x n}+\lambda_{y n}\right)\left(\rho^{r}-1\right)} \sqrt{\tilde{\theta}_{n}} \mathbf{K}_{1}\left(\sqrt{\tilde{\theta}_{n}}\right)\right)^{N}}{\log \rho}
\end{aligned}
$$

Recall that $\lambda_{x n}=\frac{d_{1 R_{n}}^{\alpha}}{2 \rho}$ and $\lambda_{y n}=\frac{d_{2 R_{n}}^{\alpha}}{\rho}$, which results $\tilde{\theta}_{n}=4 \frac{d_{1 R_{n}}^{\alpha} d_{R_{n}}^{\alpha}}{\rho^{2}}\left(\rho^{r}-1\right)\left(2\left(\rho^{r}-1\right)+1\right)$. From the definition of the multiplexing gain, we can have that $r \leq 1$, which means that $\tilde{\theta}_{n} \rightarrow 0$ for $\rho \rightarrow \infty$. So again using the property of the bessel function, we can obtain

$$
\begin{aligned}
d(r) & =-\lim _{\rho \rightarrow \infty} \frac{\log \left(1-e^{-2\left(\lambda_{x n}+\lambda_{y n}\right)\left(\rho^{r}-1\right)}\right)^{N}}{\log \rho} \\
& =-\lim _{\rho \rightarrow \infty} \frac{\log \left(\rho^{r-1}\right)^{N}}{\log \rho} \\
& =N(1-r) .
\end{aligned}
$$

And the proof for the corollary is completed.

Proof for Lemma 4: Recall that the CDF of the variable $z$ can be written as

$$
P(\mathbf{z}<z)=1-e^{-\left(\lambda_{x}+\lambda_{y}\right) z} \sqrt{4 \lambda_{x} \lambda_{y} z(z+1)} \mathbf{K}_{1}\left(\sqrt{4 \lambda_{x} \lambda_{y} z(z+1)}\right) .
$$

First of all, the upper and lower bounds of the bessel function are found. Recall from 8.432 .3 in [19], the modified bessel function of the second kind can have the following integral representation

$$
\mathbf{K}_{1}(z)=\frac{z \Gamma\left(\frac{1}{2}\right)}{2 \Gamma\left(\frac{3}{2}\right)} \int_{1}^{\infty} e^{-z t}\left(t^{2}-1\right)^{1 / 2} d t, \quad[z \geq 0]
$$


where $\Gamma(x)$ denotes the Gamma function. Since $t \geq 0$, we can have the following inequality

$$
\begin{aligned}
\mathbf{K}_{1}(z) & \geq \frac{z \Gamma\left(\frac{1}{2}\right)}{2 \Gamma\left(\frac{3}{2}\right)} \int_{1}^{\infty} e^{-z t}(t-1) d t \\
& =e^{-z} \int_{0}^{\infty} e^{-z y} d y \\
& =\frac{e^{-z}}{z} .
\end{aligned}
$$

The upper bound of the bessel function can be found from its another integral representation from 8.432.6 in [19] as

$$
\mathbf{K}_{1}(z)=\frac{z}{4} \int_{0}^{\infty} \frac{e^{-t-\frac{z^{2}}{4 t}}}{t^{2}} d t, \quad[z \geq 0] .
$$

Since $t \geq 0$, we can have $e^{-t} \leq 1$, and hence

$$
\begin{aligned}
\mathbf{K}_{1}(z) & \leq \frac{z}{4} \int_{0}^{\infty} \frac{e^{-\frac{z^{2}}{4 t}}}{t^{2}} d t \\
& =\frac{1}{z} .
\end{aligned}
$$

Combining (52) and (54), the modified bessel function of the second kind can be bounded as

$$
\frac{e^{-z}}{z} \leq \mathbf{K}_{1}(z) \leq \frac{1}{z}
$$

Applying the two bounds to the expression in (50), we can have

$$
\begin{aligned}
1-e^{-\left(\lambda_{x}+\lambda_{y}\right) z} \leq P(\mathbf{z}<z) & \leq 1-e^{-\left(\lambda_{x}+\lambda_{y}\right) z} e^{-\sqrt{4 \lambda_{x} \lambda_{y} z(z+1)}} \\
& \leq 1-e^{-\left(\lambda_{x}+\lambda_{y}+2 \sqrt{\lambda_{x}} \sqrt{\lambda_{y}}\right) z} .
\end{aligned}
$$

And the lemma is proved.

Proof for Theorem 5: Recall that the the mutual information $\mathcal{I}_{N C D}$ can be written as

$$
\mathcal{I}_{N C D}=\log \left[1+\frac{1}{2} z_{(N)}\right]
$$

where $z_{(N)}$ is the largest value of the $N$ variables $z_{n}=\frac{x_{n} y_{n}}{x_{n}+y_{n}+1}$. By using the order statistics and Lemma 4, the $\mathrm{CDF}$ of $z_{(N)}$ can be bounded as

$$
\left(1-e^{-\left(\lambda_{x}+\lambda_{y}\right) z}\right)^{N}<P\left(\mathbf{z}_{(N)}<z\right)<\left(1-e^{-\left(\sqrt{\lambda_{x}}+\sqrt{\lambda_{y}}\right)^{2} z}\right)^{N} .
$$

Hence the ergodic capacity of the proposed network coding protocol can be bounded as

$$
f\left(2\left(\sqrt{\lambda_{x}}+\sqrt{\lambda_{y}}\right)^{2}\right) \leq \mathcal{C}_{N C D} \leq f\left(2\left(\lambda_{x}+\lambda_{y}\right)\right)
$$


where $f(\cdot)$ is defined as

$$
f(\lambda)=\int_{0}^{\infty} N \lambda e^{-\lambda z}\left(1-e^{-\lambda z}\right)^{N-1} \log (1+z) d z .
$$

The justification from (58) to (59) will be provided after the proof for Theorem 5. Define $z=y-1$ and use the binomial coefficients to have

$$
\begin{aligned}
f(\lambda) & =\log e N \lambda \sum_{k=0}^{N-1} C_{N-1}^{k}(-1)^{k} e^{\lambda(k+1)} \int_{1}^{\infty} e^{-\lambda(k+1) y} \ln y d y \\
& =\log e N \lambda \sum_{k=0}^{N-1} C_{N-1}^{k}(-1)^{k} e^{\mu}\left[-\frac{1}{\mu} \mathbf{E}_{\mathbf{i}}(-\mu)\right]
\end{aligned}
$$

where $\mu=\lambda(k+1), \mathbf{E}_{\mathbf{i}}(\cdot)$ denotes the exponential-integral function and the second equality follows from Eq. 4.331.2 in [19]. Recall that the exponential-integral function can have the following series representation

$$
\mathbf{E}_{\mathbf{i}}(x)=\mathbf{C}+\ln (-x)+\sum_{k=1}^{\infty} \frac{x^{k}}{k \cdot k !},
$$

which means that the exponential-integral function can be approximated as

$$
\mathbf{E}_{\mathbf{i}}(x)=\mathbf{C}+\ln (-x), \quad \text { for } \quad x \rightarrow-0
$$

Recall that both $2\left(\lambda_{x}+\lambda_{y}\right)$ and $2\left(\sqrt{\lambda_{x}}+\sqrt{\lambda_{y}}\right)^{2}$ will be close to zero for large SNR, which results the following approximation

$$
f(\lambda) \approx \log e N \sum_{k=0}^{N-1} C_{N-1}^{k}(-1)^{k+1} \frac{\ln e^{\mathbf{C}}[\lambda(k+1)]}{k+1} .
$$

where the approximation of $e^{-x} \underset{x \rightarrow 0}{\approx} 1-x$ has also be applied. After some mathematics manipulations, the expression of the function $f(\lambda)$ can be written as

$$
\begin{aligned}
f(\lambda) & \approx \log e \sum_{k=0}^{N-1} C_{N}^{k+1}(-1)^{k+1} \ln \left[e^{\mathbf{C}} \lambda(k+1)\right] \\
& =\log e \sum_{k=0}^{N-1} C_{N}^{k+1}(-1)^{k+1} \ln \left[e^{\mathbf{C}}(k+1)\right]+\ln \lambda \log e \sum_{k=0}^{N-1} C_{N}^{k+1}(-1)^{k+1} .
\end{aligned}
$$

It is surprising to find that the summary $\sum_{k=0}^{N-1} C_{N}^{k+1}(-1)^{k+1}$ can be simplified as the following

$$
\begin{aligned}
\sum_{k=0}^{N-1} C_{N}^{k+1}(-1)^{k+1} & =\sum_{i=k+1}^{N} C_{N}^{i}(-1)^{i} \\
& =\sum_{i=0}^{N} C_{N}^{i}(-1)^{i}-1 \\
& =(1-1)^{N}-1=1 .
\end{aligned}
$$


Hence by using this result, we can have

$$
f(\lambda) \approx \sum_{k=0}^{N-1} C_{N}^{k+1}(-1)^{k+1} \log (k+1)-\mathbf{C} \log e-\log \lambda .
$$

Combining (59) and (67), the theorem can be proved.

Justification for (59): Combining (57) and (58), the CDF of the mutual information for the proposed protocol can be written as

$$
\left(1-e^{-2\left(\lambda_{x}+\lambda_{y}\right)\left(2^{z}-1\right)}\right)^{N}<P\left(\mathcal{I}_{N C D}<z\right)<\left(1-e^{-2\left(\sqrt{\lambda_{x}}+\sqrt{\lambda_{y}}\right)^{2}\left(2^{z}-1\right)}\right)^{N} .
$$

Without losing generality, we only focus on the first inequality in (68). Define $F_{1}(x)=P\left(\mathcal{I}_{N C D}<x\right)$, $F_{2}(x)=\left(1-e^{-2\left(\lambda_{x}+\lambda_{y}\right)\left(2^{z}-1\right)}\right)^{N}$ and $f_{i}(x)$ denotes the PDF function of $F_{i}(x)$. The justification of (59) is equivalent to prove

$$
\int_{0}^{\infty} x f_{1}(x) d x<\int_{0}^{\infty} x f_{2}(x) d x
$$

provided that $F_{1}(x) \geq F_{2}(x)$. The proof for this claim requires the following property of the two PDF functions. There is only one unique solution for the equation $f_{1}(x)-f_{2}(x)=0$ for $x \in(0, \infty)$, denoted as $x^{*}$. Although currently we are not able to find the formal proof for this property, simulations show that it is the case. Generally the two addressed PDF functions can be shown as in Figure 9. Since $F_{1}(x) \geq F_{2}(x)$, hence we have $f_{1}(x)-f_{2}(x) \leq 0$ for $x \in\left(0, x^{*}\right]$ and $f_{1}(x)-f_{2}(x) \geq 0$ for $x \in\left[x^{*}, \infty\right)$. Rewrite (69) as

$$
\begin{aligned}
\int_{0}^{\infty} x\left(f_{1}(x)-f_{2}(x)\right) d x & =\int_{0}^{x^{*}} x\left[f_{1}(x)-f_{2}(x)\right] d x+\int_{x^{*}}^{\infty} x\left[f_{1}(x)-f_{2}(x)\right] d x \\
& \leq x^{*} \int_{0}^{x^{*}}\left[f_{1}(x)-f_{2}(x)\right] d x+x^{*} \int_{x^{*}}^{\infty}\left[f_{1}(x)-f_{2}(x)\right] d x .
\end{aligned}
$$

It is interesting to find that $\int_{0}^{x^{*}}\left[f_{1}(x)-f_{2}(x)\right] d x$ will be the area for the bounded part $A$ shown in Figure 9 and $\int_{x^{*}}^{\infty}\left[f_{2}(x)-f_{1}(x)\right] d x$ will be the area for the part $B$. Due to the constraint that $\int_{0}^{\infty} f_{1}(x) d x=$ $\int_{0}^{\infty} f_{2}(x) d x=1$, it can be obtained that the area for the part $A$ is equal to the one for $B$. Using such equality, (70) can be written as

$$
\int_{0}^{\infty} x\left(f_{1}(x)-f_{2}(x)\right) d x \leq x^{*} \times 0=0,
$$

and the claim in (69) is proved. 


\section{REFERENCES}

[1] S. Zhang, S. Liew, and P. Lam, "Physical layer network coding," in Proc. 12th Annual International Conference on Mobile Computing and Networking (ACM MobiCom 2006), Sept. 2006.

[2] R. Ahlswede, N. Cai, S. R. Li, and R. W. Yeung, "Network information flow," IEEE Trans. Information Theory, vol. 46, pp. 1204-1217, Jul. 2000.

[3] R. W. Yeung, S.-Y. R. Li, N. Cai, and Z. Zhang, Network Coding Theory, Foundations and Trends in Communications and Information Theory. Now Publishers Inc., 2006.

[4] S. Katti, H. Rahul, W. Hu, D. Katabi, M. Medard, and J. Crowcroft, "Xors in the air: Practical wireless network coding," in Proc. ACM SIGCOMM, Sept. 2006, pp. 243-254.

[5] J. Liu, D. Goeckel, and D. Towsley, "Bounds on the gain of network coding and broadcasting in wireless networks," to be presented at IEEE INFOCOM, May 2007.

[6] Y. Chen, S. Kishore, and J. Li, "Wireless diversity through network coding," in Proceeding of IEEE Wireless Communications and Networking Conference (WCNC), Mar. 2006, pp. 1681 - 1686.

[7] Y. Hao, D. Goeckel, Z. Ding, D. Towsley, and K. Leung, “Achievable rates for network coding on the exchange channel,” Proceeding of the IEEE Military Communications Conference, pp. 371-382, Oct. 2007.

[8] Y. Wu, P. Chou, and S. Kung, "Information exchange in wireless networks with network coding and physical layer broadcast," Technical Report MSF-TR-2004-78, Microsoft Research, Aug. 2004.

[9] T. S. Rappaport, Wireless communications: principles and practice. Prentice Hall, 1998.

[10] S. Zhu and K. K. Leung, "Distributed cooperative routing for uwb ad-hoc networks," to be presented at the IEEE International Conference on Communications (ICC07), June 2007.

[11] J. N. Laneman, D. N. C. Tse, and G. W. Wornell, "Cooperative diversity in wireless networks: Efficient protocols and outage behavior," IEEE Trans. Information Theory, vol. 50, pp. 3062-3080, Dec. 2004.

[12] Z. Ding, T. Ratnarajah, and C. Cowan, "On the diversity-multiplexing tradeoff for wireless cooperative multiple access systems," to appear in IEEE Trans. Signal Processing, pp. 4627-4638, Sept. 2007.

[13] J. Proakis, Digital Communications, 4th ed. McGraw-Hill, New York, 2000.

[14] C.-J. Chen and L.-C. Wang, "Enhancing coverage and capacity for multiuser MIMO system by utilizing scheduling," IEEE Trans. on Wireless Communications, vol. 5, pp. 1148-1157, May 2006.

[15] S. Cui and A. J. Goldsmith, "Cross-layer design in energy-constrained networks using cooperative MIMO techniques," EURASIP/Elsevier Signal Processing Journal, vol. 86, pp. 425-436, Aug. 2006.

[16] L. Zheng and D. N. C. Tse, "Diversity and multiplexing : a fundamental tradeoff in multiple antenna channels," IEEE Trans. Information Theory, vol. 49, pp. 1073-1096, May 2003.

[17] D. N. C. Tse, P. Viswanath, and L. Zheng, "Diversity-multiplexing tradeoff in multiple-access channels," IEEE Trans. Information Theory, vol. 50, pp. 1859-1874, Sept. 2004.

[18] Y. Zhao, R. Adve, and T. J. Lim, "Outage probability at arbitrary SNR with cooperative diversity," IEEE Comm. Letter, vol. 9, pp. 700-702, Aug. 2005.

[19] I. S. Gradshteyn and I. M. Ryzhik, Table of Integrals, Series and Products, 6th ed. Academic Press, 2000.

[20] H. A. David and H. N. Nagaraja, Order Statistics. John Wiley,Third Edition, 2003. 


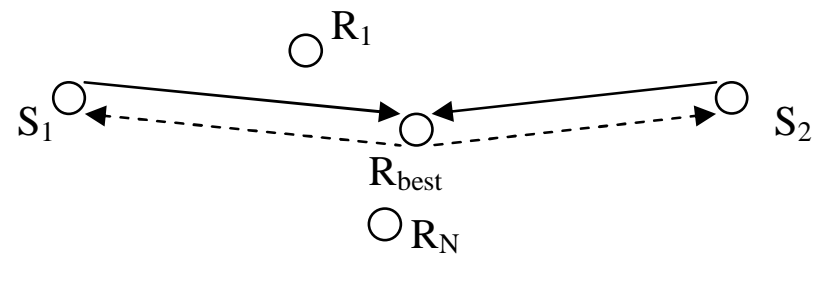

Time slot 1

Time slot 2

Fig. 1. A diagram for the addressed information exchanging scenario and the transmission strategy for the proposed network coding with diversity.

\section{TABLE I}

PERFoRMANCE LOSS DUE TO THE USE OF A SUBOPTIMAL RELAY

\begin{tabular}{|l|l|l|l|l|l|}
\hline $\mathcal{C} \backslash S N R$ & 5 & 10 & 15 & 20 & 25 \\
\hline $\mathcal{C}_{21, \text { optimal }}, N=2$ & 0.2580 & 0.6899 & 1.5452 & 2.7370 & 4.2098 \\
$\mathcal{C}_{21, \text { suboptimal }}, N=2$ & 0.2549 & 0.6744 & 1.5115 & 2.6687 & 4.1068 \\
\hline $\mathcal{C}_{21, \text { optimal }}, N=10$ & 0.8953 & 1.9070 & 3.2431 & 4.8207 & 6.4140 \\
$\mathcal{C}_{21, \text { suboptimal }}, N=10$ & 0.8722 & 1.8572 & 3.1434 & 4.7216 & 6.2969 \\
\hline
\end{tabular}

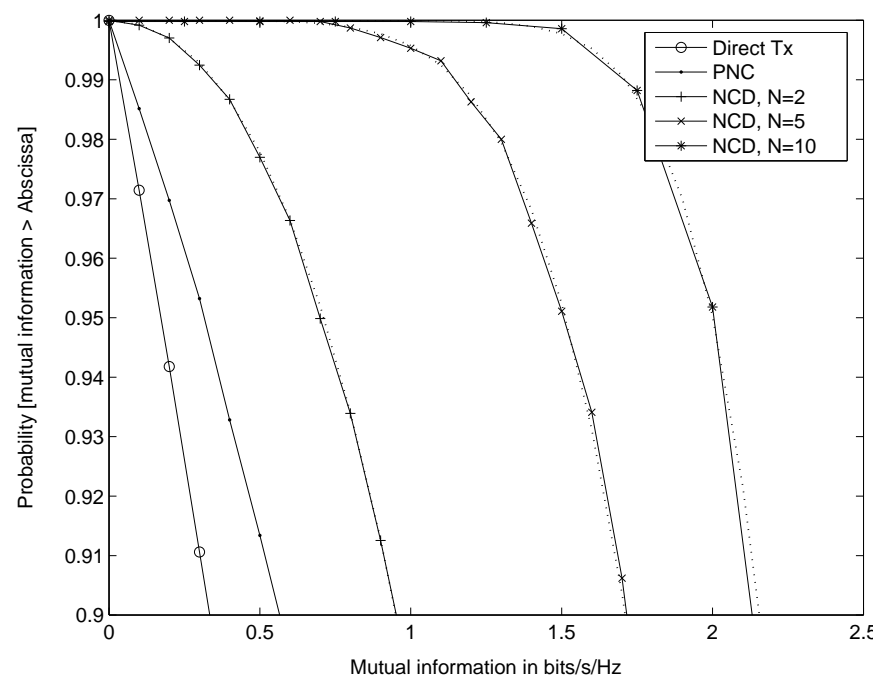

(a) $\mathrm{SNR}=10 \mathrm{~dB}$

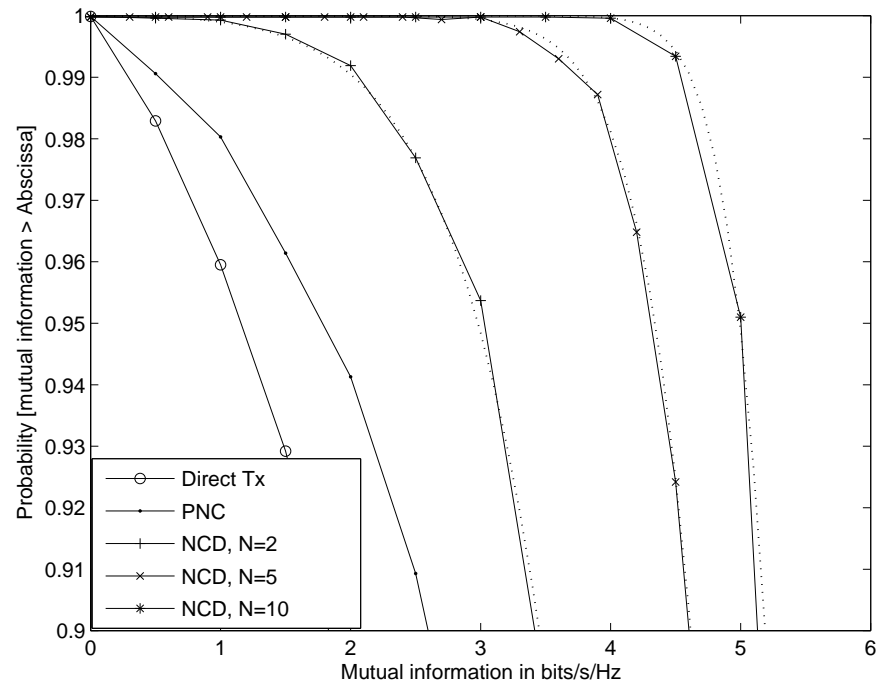

(b) $\mathrm{SNR}=20 \mathrm{~dB}$

Fig. 2. Mutual information complementary cumulative distribution functions. The distance of the two sources is $2 \mathrm{~m}$. Solid line represents the results obtained by using the Monte-Carlo simulations, and the dotted line represents the results calculated by using the proposed analytical formulations.

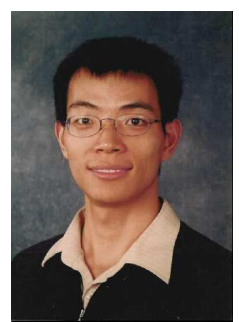

Zhiguo Ding (S'03-M'05) received his B.Eng in Electrical Engineering from the Beijing University of Posts and Telecommunications in 2000, and the Ph.D degree in Electrical Engineering from Imperial College London in 2005.

From 2000 to 2002, he was a soft engineer in Datang Telecom Inc. and Vimicro Corporation, Beijing, P.R.China. From Jul. 2005 to Sept. 2007, he was with Queen's University Belfast and Imperial College as a postdoctoral researcher. Since Oct. 2008, he has been with the Department of Communication Systems, Lancaster University, as a Lecturer. His research interests are cross-layer optimization, cooperative diversity, statistical signal processing and information 


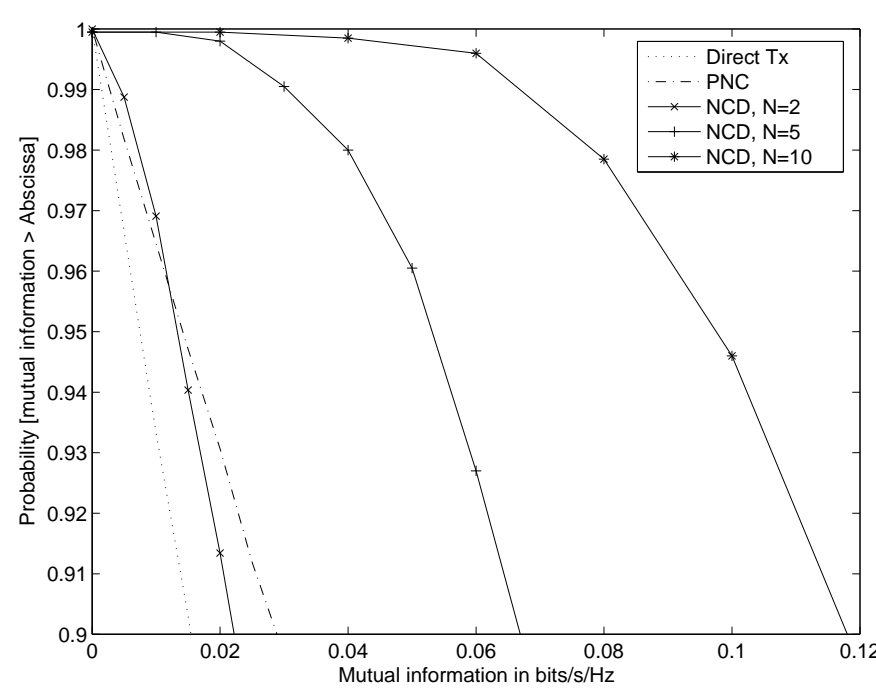

(a) $\mathrm{SNR}=10 \mathrm{~dB}$

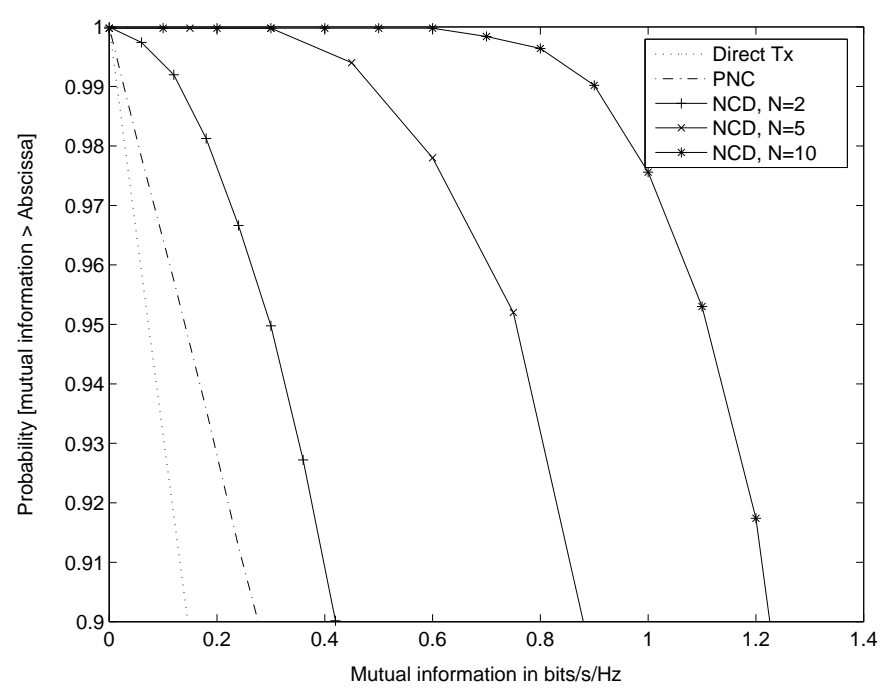

(b) $\mathrm{SNR}=20 \mathrm{~dB}$

Fig. 3. Mutual information complementary cumulative distribution functions. The distance of the two sources is $10 \mathrm{~m}$. Solid line represents the proposed network coding scheme, dot-dot line represents direct transmission and dash-dash line represents the physical network coding.

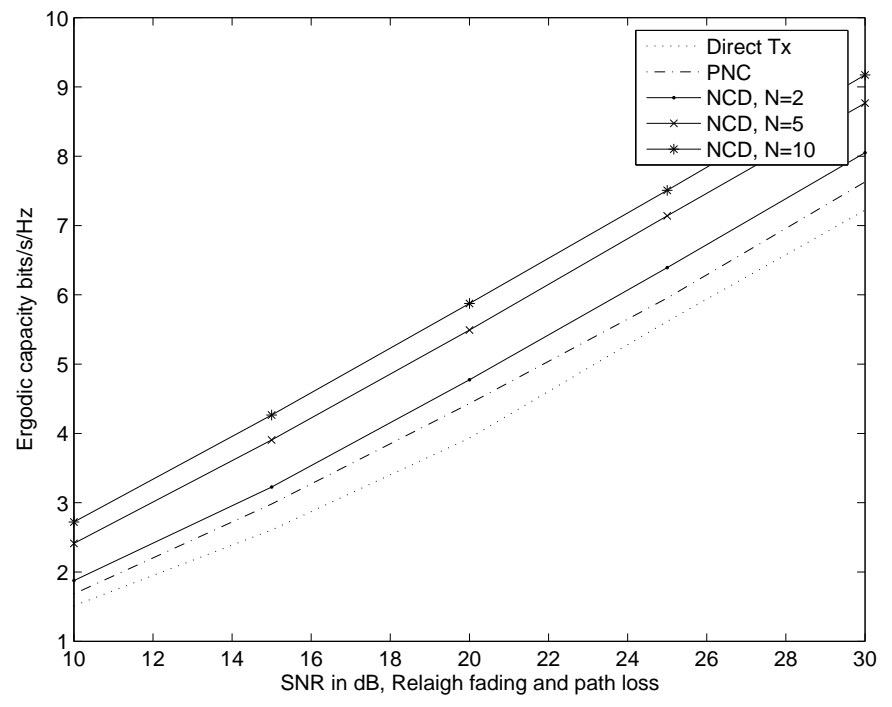

(a) $d_{12}=2 m$

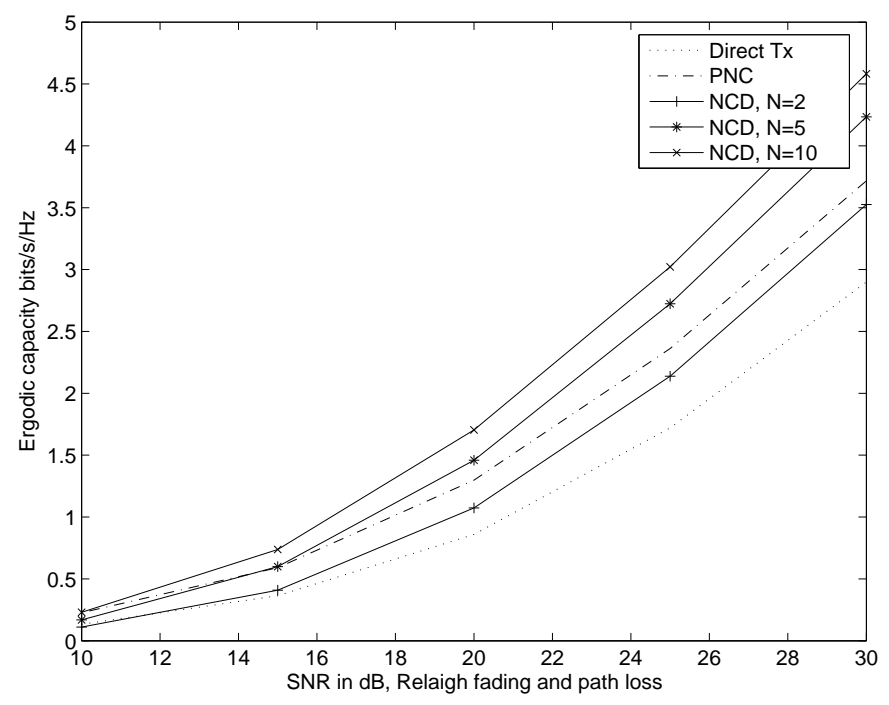

(b) $d_{12}=10 \mathrm{~m}$

Fig. 4. Ergodic capacity vs SNR. Solid line represents the proposed network coding scheme, dot-dot line represents direct transmission and dash-dash line represents the physical network coding.

theory. 


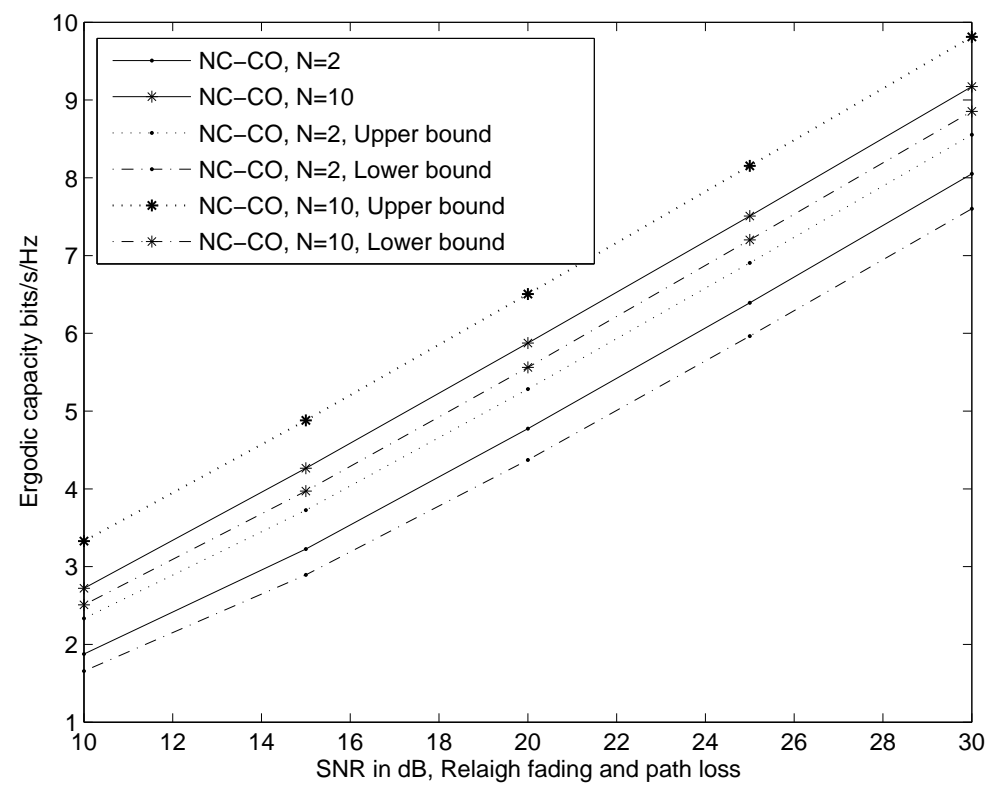

Fig. 5. Ergodic capacity vs SNR. The distance of the two sources is $2 \mathrm{~m}$. Solid lines represent the results obtained by using the Monte-Carlo simulations. The dotted lines represent the upper bound and the dash lines represent the lower bound calculated by using Theorem 2 .

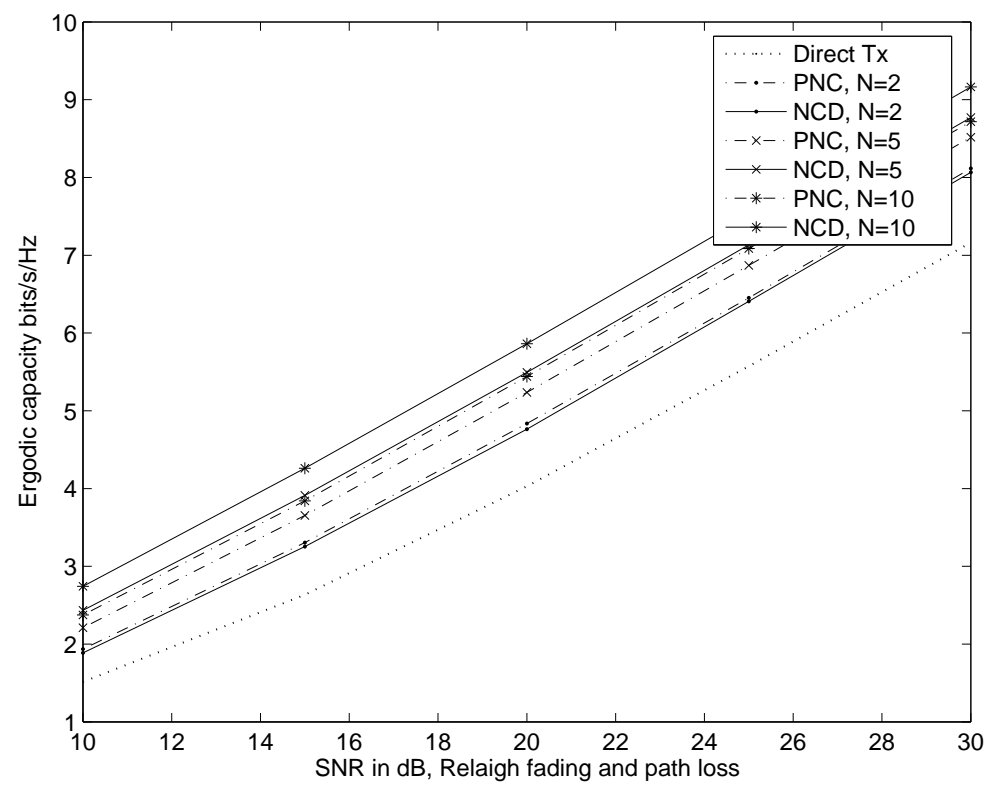

Fig. 6. Ergodic capacity vs SNR. The distance of the two sources is $2 \mathrm{~m}$. Solid line represents the proposed network coding scheme, dot-dot line represents direct transmission and dash-dash line represents the physical network coding.

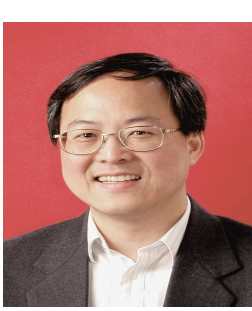

Kin K. Leung received his B.S. degree (with first-class honors) from the Chinese University of Hong Kong in 1980, and his M.S. and Ph.D. degrees in computer science from University of California, Los Angeles, in 1982 and 1985 , respectively.

He started his career at AT\&T Bell Labs in 1986 and worked at its successor companies, AT\&T Labs and Bell Labs of Lucent Technologies, until 2004. Since then, he has been the Tanaka Chair Professor in Internet Technology at Imperial College in London. His research interests include network resource allocation, MAC protocol, TCP/IP protocol, distributed optimization algorithms, mobility management, network architecture, real-time applications and teletraffic issues for 


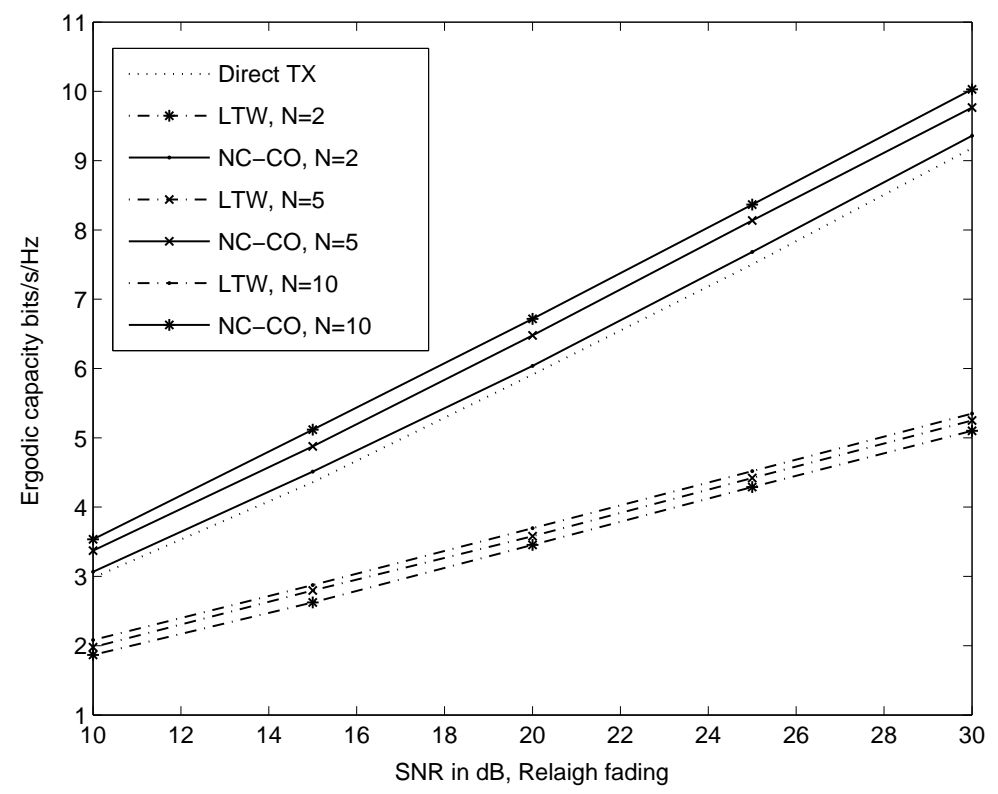

Fig. 7. Ergodic capacity vs SNR. Solid lines represent the results for the proposed cooperative protocol, the dashed line denotes the results for the LTW scheme, and the dotted line represents for the direct transmission scheme.

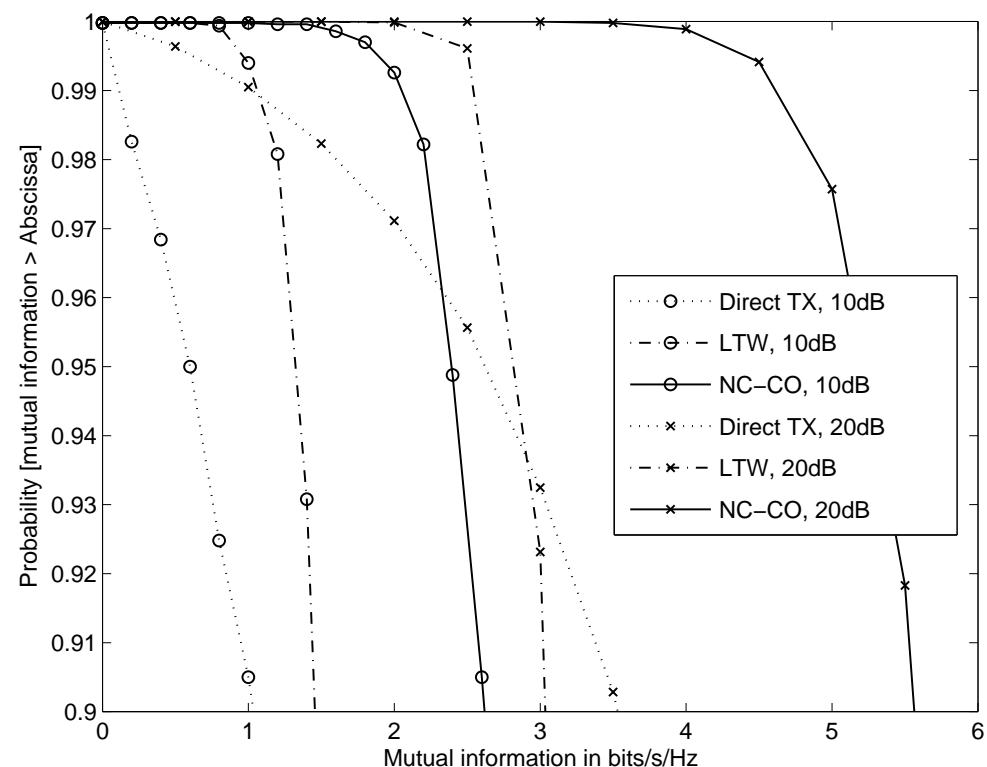

Fig. 8. Mutual information complementary cumulative distribution functions. Solid lines represent the results for the proposed cooperative protocol, the dashed line denotes the results for the LTW scheme, and the dotted line represents for the direct transmission scheme.

broadband wireless networks, wireless sensor and ad-hoc networks. He is also interested in a wide variety of wireless technologies, including IEEE 802.11, 802.16, and 3G and future generation cellular networks.

He received the Distinguished Member of Technical Staff Award from AT\&T Bell Labs in 1994, and was a co-recipient of the 1997 Lanchester Prize Honorable Mention Award. He was elected in 2001 as an IEEE Fellow for his contributions to performance analysis, protocol design, and control algorithms for communications networks. He receives the Royal Society Wolfson Research Merits Award from 2004 to 2009. He has published widely and acquired patents in many areas of communication networks. He has actively served on many conference committees, including as the committee co-chair for the Multiaccess, Mobility and Teletraffic for Wireless Communications 


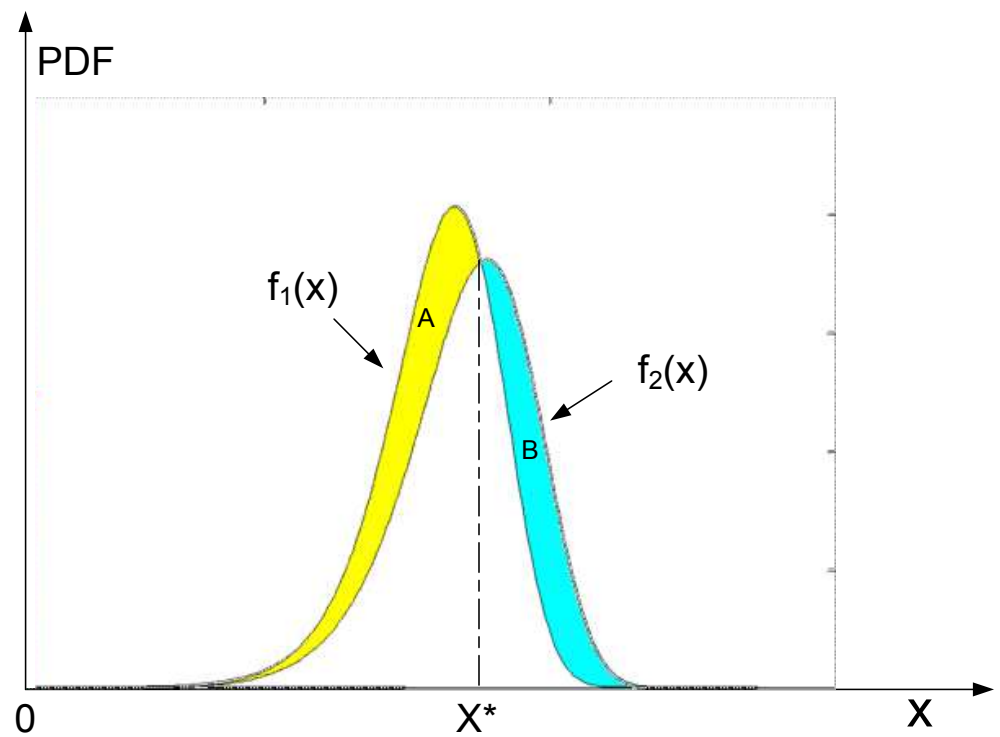

Fig. 9. The PDF function for the two variables.

(MMT'98), the Wireless Networking Theory Symposium of IEEE ICC 2002 and the First Annual Conference of International Technology Alliance (ITA) 2007 (sponsored by the U.S. Army and U.K. Ministry of Defence). He was a guest editor for the IEEE Journal on Selected Areas in Communications (JSAC), IEEE Wireless Communications and the MONET journal, and as an editor for the JSAC: Wireless Series. Currently, he is an editor for the IEEE Transactions on Communications, the Transactions on Wireless Communications and International Journal on Sensor Networks.

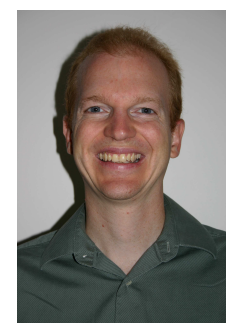

Dennis Goeckel split time between Purdue University and Sundstrand Corporation from 1987-1992, receiving his BSEE from Purdue in 1992. From 1992-1996, he was a National Science Foundation Graduate Fellow and then Rackham Pre-Doctoral Fellow at the University of Michigan, where he received his MSEE in 1993 and his Ph.D. in 1996, both in Electrical Engineering with a specialty in Communication Systems. In September 1996, he joined the Electrical and Computer Engineering department at the University of Massachusetts, where he is currently a Professor. His current research interests are in the areas of communication systems and wireless network theory.

Dr. Goeckel was the recipient of a 1999 CAREER Award from the National Science Foundation for "Coded Modulation for HighSpeed Wireless Communications". He was a Lilly Teaching Fellow at UMass-Amherst for the 2000-2001 academic year and received the University of Massachusetts Distinguished Teaching Award in 2007. He served as an Editor for the IEEE Journal on Selected Areas in Communications: Wireless Communication Series during its transition to the IEEE Transactions on Wireless Communications from 19992002, and as a Technical Program Committee Co-Chair for the Communication Theory Symposium at IEEE Globecom 2004. He is currently an Editor for the IEEE Transactions on Communications and a Co-Chair for the Wireless Communications Symposium at IEEE Globecom 2008 . 
Don Towsley holds a B.A. in Physics (1971) and a Ph.D. in Computer Science (1975) from University of Texas. He

PLACE

PHOTO

HERE is currently a Distinguished Professor at the University of Massachusetts in the Department of Computer Science. He has held visiting positions at IBM T.J. Watson Research Center, Yorktown Heights, NY; Laboratoire MASI, Paris, France; INRIA, Sophia-Antipolis, France; AT\&T Labs - Research, Florham Park, NJ; and Microsoft Research Lab, Cambridge, UK. His research interests include networks and performance evaluation.

He currently serves as Editor-in-Chief of IEEE/ACM Transactions on Networking, and on the editorial boards of Journal of the ACM and IEEE Journal on Selected Areas in Communications and has previously served on numerous other editorial boards. He was Program Co-chair of the joint ACM SIGMETRICS and PERFORMANCE ' 92 conference and the Performance 2002 conference. $\mathrm{He}$ is a member of ACM and ORSA.

He has received the 2007 IEEE Koji Kobayashi Award, the 2007 ACM SIGMETRICS Achievement Award, the 1998 IEEE Communications Society William Bennett Best Paper Award, and numerous conference/workshop best paper awards. Last, he has been elected Fellow of both the ACM and IEEE. 